\title{
Tidal evolution of close-in extra-solar planets
}

\author{
Brian Jackson $^{1}$, Richard Greenberg ${ }^{1}$ and Rory Barnes ${ }^{1}$ \\ ${ }^{1}$ Lunar and Planetary Laboratory, University of Arizona, \\ 1629 E University Blvd \\ Tucson, Arizona 85721-0092 USA \\ email: bjackson@lpl.arizona.edu
}

\begin{abstract}
The distribution of eccentricities $e$ of extra-solar planets with semi-major axes $a>$ $0.2 \mathrm{AU}$ is very uniform, and values for $e$ are generally large. For $a<0.2 \mathrm{AU}$, eccentricities are much smaller (most $e<0.2$ ), a characteristic widely attributed to damping by tides after the planets formed and the protoplanetary gas disk dissipated. We have integrated the classical coupled tidal evolution equations for $e$ and $a$ backward in time over the estimated age of each planet, and confirmed that the distribution of initial $e$ values of close-in planets matches that of the general population for reasonable tidal dissipation values $Q$, with the best fits for stellar and planetary $Q$ being $\sim 10^{5.5}$ and $\sim 10^{6.5}$, respectively. The current small values of $a$ were only reached gradually due to tides over the lifetimes of the planets, i.e., the earlier gas disk migration did not bring all planets to their current orbits. As the orbits tidally evolved, there was substantial tidal heating within the planets. The past tidal heating of each planet may have contributed significantly to the thermal budget that governed the planet's physical properties, including its radius, which in many cases may be measured by observing transit events. Here we also compute the plausible heating histories for a few planets with anomalously large measured radii, including HD 209458 b. We show that they may have undergone substantial tidal heating during the past billion years, perhaps enough to explain their large radii. Theoretical models of exoplanet interiors and the corresponding radii should include the role of large and time-variable tidal heating. Our results may have important implications for planet formation models, physical models of "hot Jupiters", and the success of transit surveys.
\end{abstract}

Keywords. celestial mechanics, planetary systems: formation, protoplanetary disks

\section{Introduction}

As the number of known extra-solar planetary orbits has increased, interesting patterns are emerging. Figure 1 shows the semi-major axes $a$ and eccentricities $e$. Eccentricities of extra-solar planets with $a>0.2$ AU average 0.3 and are broadly distributed up to near 1. The distribution of eccentricities is fairly uniform over $a$. For example, a KolmogorovSmirnov (K-S) test shows that the $e$ distribution for $a$ between 0.2 and 1.0 AU matches that for $a$ between 1.0 and $5.0 \mathrm{AU}$ at the $96 \%$ confidence level. However, for close-in extrasolar planets (by which we mean $a<0.2 \mathrm{AU}$ ), $e$ values are smaller, with an average of 0.09 , but still large compared to our solar system. For $a<0.2 \mathrm{AU}$, the K-S test shows agreement at only the $0.1 \%$ level compared with planets further out.

Here we consider the conventional idea that close-in planets began with a distribution of $e$ similar to that of planets farther out. Because the magnitude of tidal effects falls off very rapidly with increasing $a$, Rasio et al. 1996 suggested that tides could have reduced $e$ for close-in planets and not for those farther out.

The implications of this hypothesis for planetary masses $M_{p}$, radii $R_{p}$, and heating rates has been considered in previous studies (Rasio et al. 1996; Trilling 2000; Bodenheimer et al. 2003). Several assumptions have been common. First, the tidal dissipation 
parameter $Q_{p}$ for the planet is usually based on model-dependent estimates based on the tidal evolution of the Jovian satellite system (Yoder \& Peale 1981) or on physical theories of tidal dissipation (Goldreich \& Nicholson 1977; Ogilvie \& Lin 2004). Second, effects of tides raised on the star are only partially taken into account. Often the orbitcircularizing effects of tides raised on the host star are ignored, although they can be important. Third, some studies (e.g. Ford \& Rasio 2006) assumed that orbital angular momentum is conserved during tidal evolution, which is not true when tides on the star are taken into account. Fourth, previous work ignored the strong coupling of the tidal evolution of $e$ with that of $a$, generally describing the circularization of an orbit in terms of an implicitly exponential "timescale". In fact, the coupling with a means that evolution is more complex than a simple exponential damping. Moreover, tidal evolution has resulted in significant inward migration of many close-in planets.

In our study, we avoid these assumptions. We use the full tidal evolution equations to determine possible past orbital change. The equations account for tides raised on the stars by the planet and on the planet by the star, each of which affects changes in both $e$ and $a$. We employ conservative assumptions about $M_{p}$ and $R_{p}$, to test the tidal

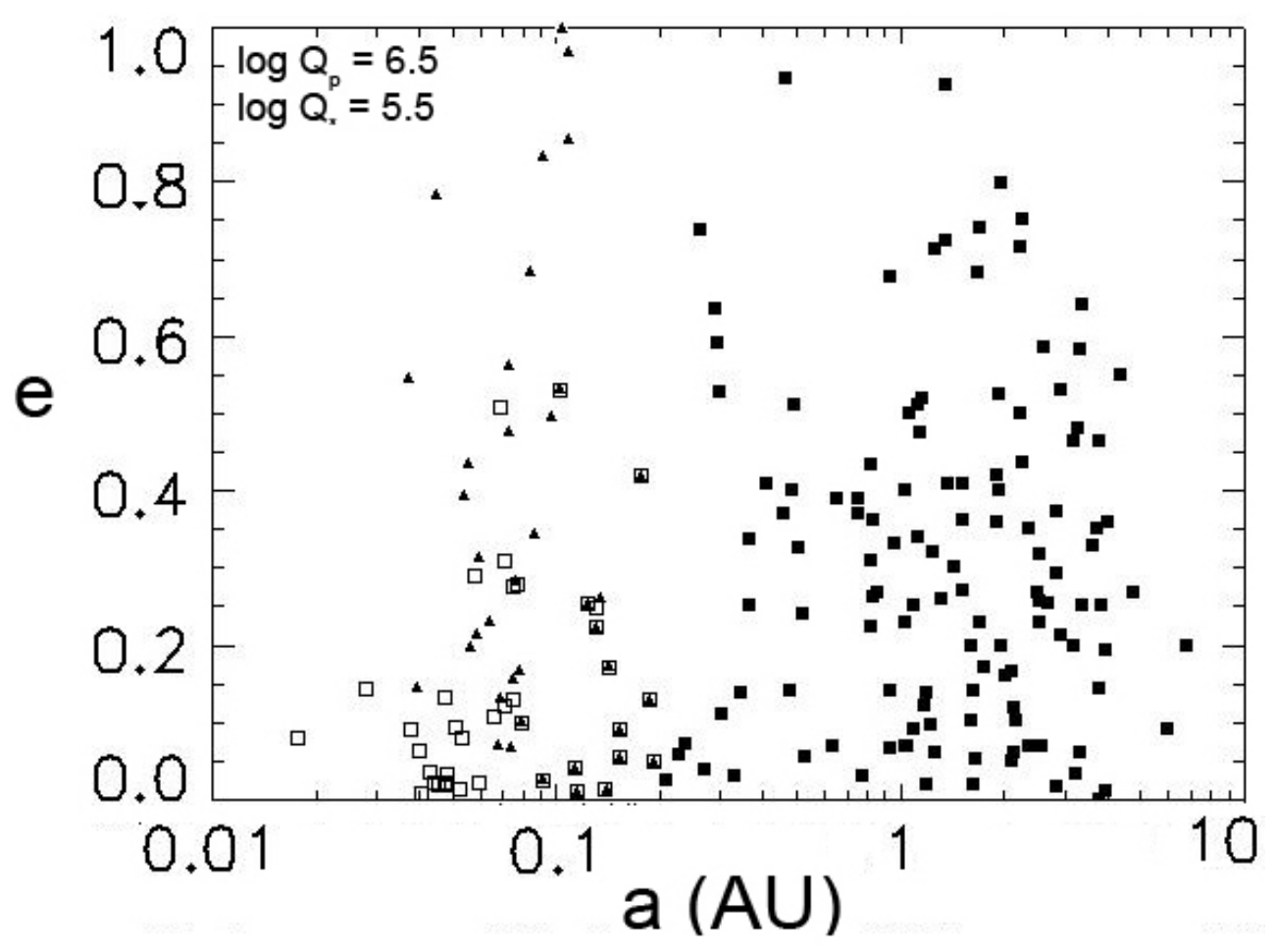

Figure 1. Distributions of orbital elements. Squares (filled and open) represent the currently observed orbital elements, with the open squares (with $a<0.2 \mathrm{AU}$ ) being candidates for significant tidal evolution. Triangles show the initial orbital elements $\left(e_{\text {initial }}\right.$ and $\left.a_{\text {initial }}\right)$ determined by integrating the equations of tidal evolution backward in time to the formation of the planet, using $Q$ values that gave the best fit of the $e$ distribution to that of the other planets. 
circularization hypothesis. And we consider a wide range of possible $Q$ values, allowing us to determine which pair of $Q_{p}$ and $Q_{*}$ values yields the most plausible evolution history.

These calculations also yield the corresponding past tidal heating history for each planet. During the course of the tidal evolution, tidal distortion of the figure of the planet can result in substantial amounts of internal heating at the expense of orbital energy, so the heating rate as a function of time is coupled to the evolution of the orbit. In a typical case, tidal heating might have begun modest, but then increased as tides reduced $a$. As the tides became stronger, they would circularize the orbit, which in turn would shut down the tidal heating mechanism. The relative strength and timing of these two effects would determine a planet's history, typically with a gradual increase in the heating rate followed by a decrease.

The thermal history of a planet is critical to determining its physical properties. For example, studies of extra-solar planets have considered the effects of heating on their radii, which can be measured directly by transit observations. Heat sources that have been considered in these models include the energy of planetary accretion and radiation from the star, as well as tidal heating (Bodenheimer et al. 2003; Mardling 2007). In many cases the theoretical predictions match the observations reasonably well (Burrows et al. 2007). However, there are notable exceptions. HD $209458 \mathrm{~b}$ has been observed by Knutson et al. 2007 to have a radius of 1.32 Jupiter radii $\left(R_{J}\right)$, which is $10-20 \%$ larger than predicted by theoretical modeling Guillot 2005. Similarly, HAT-P-1 b is $10-20 \%$ larger than predicted by theory (Bakos et al. 2007a). On the other hand, HAT-P-2 b seems to be smaller than theory would predict (Bakos et al. 2007b), while for GJ 436 b, theory predicts a radius consistent with observation (Gillon et al. 2007).

Theoretical models to date have not taken into account the history of tidal heating for close-in planets, and of course those are the planets most likely to have radii measurable by transits. The tidal heating histories reported here and in Jackson et al. 2008b provides motivation and a basis for construction of improved physical models.

\section{Method}

To test the hypothesis that tides have been responsible for reducing $e$, we numerically integrated the canonical tidal evolution equations of Goldreich \& Soter 1966 and Kaula 1968 backwards in time for all close-in planets for which we have adequate information (see Jackson et al. 2008a for details). For each planet, we began the integration with the current best estimates of $e$ and $a$ (e $e_{\text {current }}$ and $\left.a_{\text {current }}\right)$ and integrated backwards over the estimated age of the host star to find the orbital elements, $e_{\text {initial }}$ and $a_{\text {initial }}$. We assumed tidal evolution dominated the orbital evolution after the protoplanetary disk dissipated and collisional effects became negligible, which probably happened only a few Myr after the star's formation. Hence $e_{\text {initial }}$ and $a_{\text {initial }}$ may describe the orbits at that time. We repeated the integration for 289 combinations of $Q_{p}$ and $Q_{*}$, each $Q$ ranging from $10^{4}$ to $10^{8}$.

Our study involves a number of assumptions and approximations which are detailed and discussed by Jackson et al. 2008a. In particular, our model assumes host stars rotate much more slowly than their close-in companion planet revolves, an assumption largely corroborated by observation (Trilling 2000; Barnes 2001). As a result, tides raised on the star by the planet tend to decrease both $e$ and $a$, as do tides raised on the planet. Our results will inevitably need to be revisited and refined as improved data and physical models become available. Of necessity, we considered only planets for which the reported $e$ is non-zero, and for which there is some estimate available for the age of the system. Even with these restrictions, we can still study about $40 \%$ of all known close-in planets. 
Stellar masses and radii come from a variety of sources: Bakos et al. 2007a; Bakos et al. 2007b; Da Silva et al. 2006; Fischer \& Valenti 2005; Moutou et al. 2006; Saffe et al. 2006; Gorda \& Svechnikov 1996; and Takeda et al. 2007. For planetary masses, we use the radial-velocity minimum mass. For planetary radii, if $M_{p}>0.3$ Jupiter's mass, we fix $R_{p}=1.2 R_{J}$ since, for Jovian planets in this range of mass, the radius is insensitive to mass (Hubbard 1984). This value is near the average radius of almost all observed transiting extra-solar planets. For planets with $M_{p}<0.3$ Jupiter's mass, we assume the planet has Jupiter's density and scale $R_{p}$ accordingly. This assumption agrees fairly well with the observed radius for GJ 436 b (Gillon et al. 2007; Deming et al. 2007). Where $R_{p}$ and $M_{p}$ have been determined directly from transit observations, we instead use those values. Planetary data were taken from a variety of sources: Bakos et al. 2007a; Bakos et al. 2007b; Butler et al. 2006; Da Silva et al. 2006; Deming et al. 2007; Gillon et al. 2007; Johnson et al. 2006; Knutson et al. 2007; Laughlin et al. 2005; Lovis et al. 2006; McArthur et al. 2004; Maness 2007; Mayor 2004; Moutou et al. 2006; Rivera et al. 2005; Udry et al. 2002; Valenti \& Fischer 2005; Vogt et al. 2005; Wright et al. 2006; and Zucker et al. 2004.

Based upon the above model, we can also calculate the tidal heating rate for planets undergoing tidal circularization. The tidal heating of the planet results from the reduction of orbital energy (and hence $a$ ) due to energy dissipation in the planet. By tracking changes in $a$ due to tides raised on the planet throughout the process of tidal circularization, we can estimate the tidal heating rate over the whole lifetime of the planet (Peale et al. 1979). The magnitude and, indeed, the very shape of the past heating curve over time depends sensitively upon the assumed current orbital elements. Accordingly, we have calculated multiple plausible heating curves for each planet, corresponding to the range of observationally allowed values for $e_{\text {current }}$ and $a_{\text {current }}$.

\section{Orbital Evolution}

The 289 combinations of $Q_{p}$ and $Q_{*}$ that we tested gave a wide variety of distributions of initial eccentricities for the close-in planets. (Remember "initial" refers to the time, shortly after formation, that a planet's orbital evolution begins to be dominated by tides.) In addition to the current orbits (squares), Figure 1 shows the computed distributions of $e_{\text {initial }}$ and $a_{\text {initial }}$ (filled triangles) for the case of $Q_{p}=10^{6.5}$ and $Q_{*}=10^{5.5}$. We compared the $e_{\text {initial }}$ distributions for the close-in planets with the standard $e$ distribution observed for $a>0.2 \mathrm{AU}$. In this case, the agreement is excellent, with a K-S score of $90 \%$. These $Q$ values are well within plausible ranges. Reasonable fits can also be obtained with other values of $Q_{*}$ as long as $Q_{p} \sim 10^{6.5}$. Other good fits $(\mathrm{K}-\mathrm{S} \sim 70 \%)$ have $Q_{*}=10^{4.25}$ or $>10^{6.75}$.

Figure 2 shows the evolutionary track of $a$ and $e$ over time for each of the planets, in the case of the best-fit $Q$ values. The current orbital elements are at the lower left end of each trajectory in $(a, e)$ space. These points correspond to empty squares in Figure 1. The tick marks show the orbital elements at intervals of $500 \mathrm{Myr}$, going back in time, for 15 Gyr, from the present toward the upper right. Black dots have been placed at a point representing the best age estimate for the planetary system. These same points appear as the triangles in Fig. 1.

The evolutionary histories derived here include substantial changes in semi-major axis coupled with the changes in eccentricity. For many close-in planets, Figures 1 and 2 show that initial $a$ values were significantly higher than the currently observed values. These initial values of $a$ likely represent their locations at the termination of gas disk migration in each early planetary system. Given the extent of tidal migration for observed close-in 


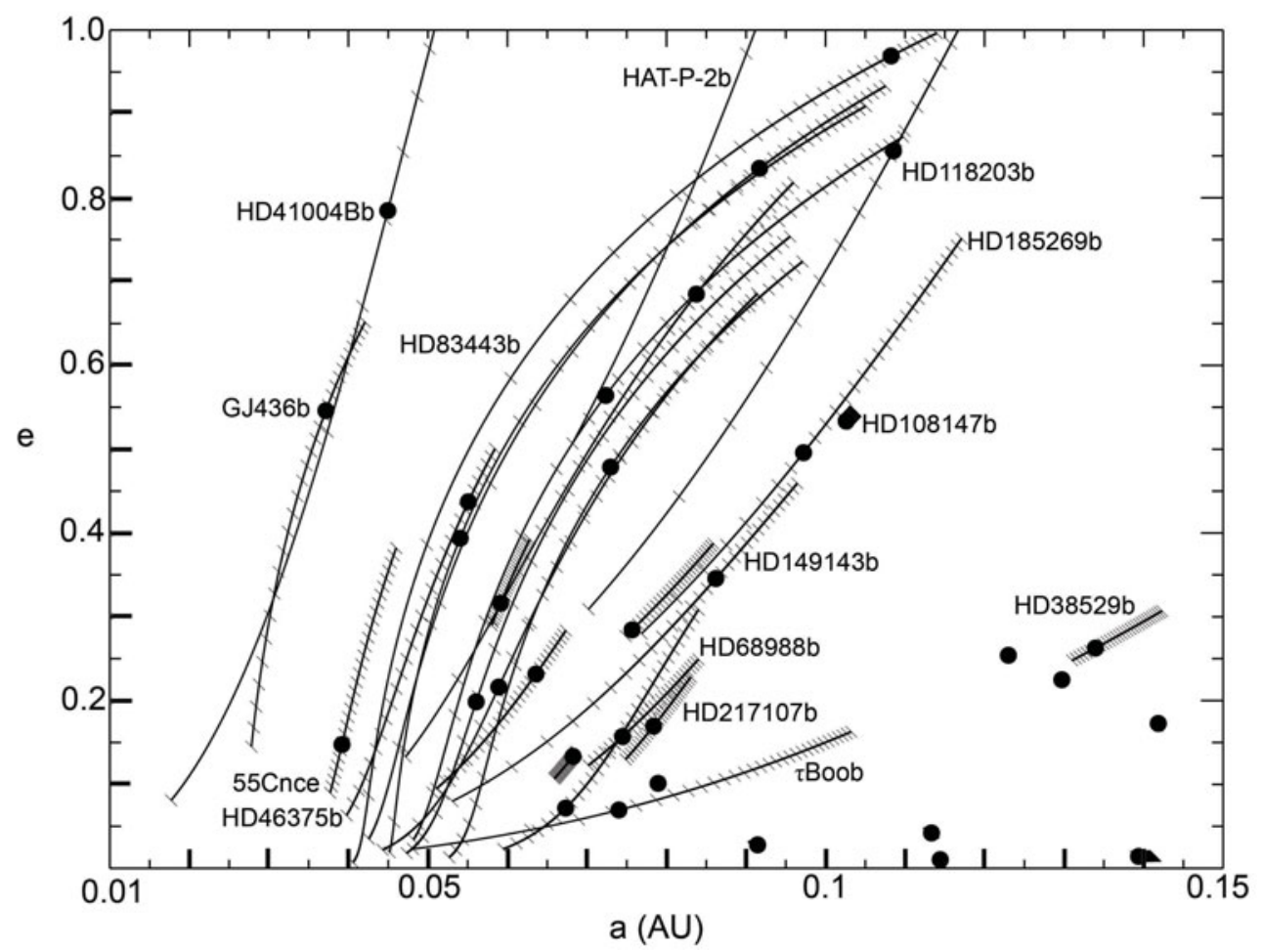

Figure 2. Tidal evolution of $e$ and $a$ for the sample of known close-in extra-solar planets using our best-fit values of $Q_{*}=10^{5.5}$ and $Q_{p}=10^{6.5}$. Solid curves represent the trajectories of orbital evolution from current orbits (lower left end of each curve) backward in time (toward the upper right). On the trajectories, tick marks are spaced every $500 \mathrm{Myr}$ to indicate the rate of tidal evolution. Tidal integrations were performed for 15 Gyr for all planets, but the filled circles indicate the initial values of orbital elements at the beginning of each planet's life. Due to space restrictions, most planets are not labeled, however they can be identified by the $(e, a)$ values at the lower left end of each trajectory. Jackson et al. 2008a includes a table of $e_{\text {current }}$ and $a_{\text {current }}$ (Table 1 ) which can be used to identify planets in this figure.

planets, planets that formed inward of $0.04 \mathrm{AU}$ could have subsequently fallen into their host stars. Consideration of such hypothesized scenarios will be the subject of future work.

\section{Tidal Heating}

Using the results of our tidal evolution calculations, we modeled the past tidal heating rates for numerous planets. In Figure 3, we illustrate plausible heating histories for three interesting examples: HD 209458 b, HAT-P-1 b and GJ $436 \mathrm{~b}$, using $Q_{p}=10^{6.5}$ and $Q_{*}$ $=10^{5.5}$. Results for other planets are presented in Jackson et al. 2008b. Note that in these examples the planetary radii have been held constant, even though the changing heating rates would most likely produce a changing radius. 
For HD 209458 b, Burrows et al. 2007 suggest that a heating rate of about $4 \times 10^{19}$ $\mathrm{W}$ would be required to yield the observed planetary radius, which is much larger than any allowable current tidal heating rate. However, the history plotted in Figure 3 shows that the required heating rate was available as recently as 1 billion years ago. If the lag in the response of the planet to the heating rate were on the order of a billion years, this heating rate may explain the observed large radius. Such a lag seems reasonable based on the long duration of the influence of heat of formation on the planet's radius in the modeling by Burrows et al. 2007.

Like HD 209458 b, HAT-P-1 b's observed radius of $1.36 R_{J}$ (Bakos et al. 2007a) is larger than expected from theoretical modeling that did not include tidal heating (Guillot 2005). Similar to HD 209458 b, Figure 3 shows that its heating rate $\sim 1$ Gyr ago was substantially higher than the present tidal heating. For both HD 209458 b and HAT-P-1 $\mathrm{b}$, the substantial heating rate $\sim 3-4 \times 10^{19} \mathrm{~W}$ about 1 Gyr ago may help account for the

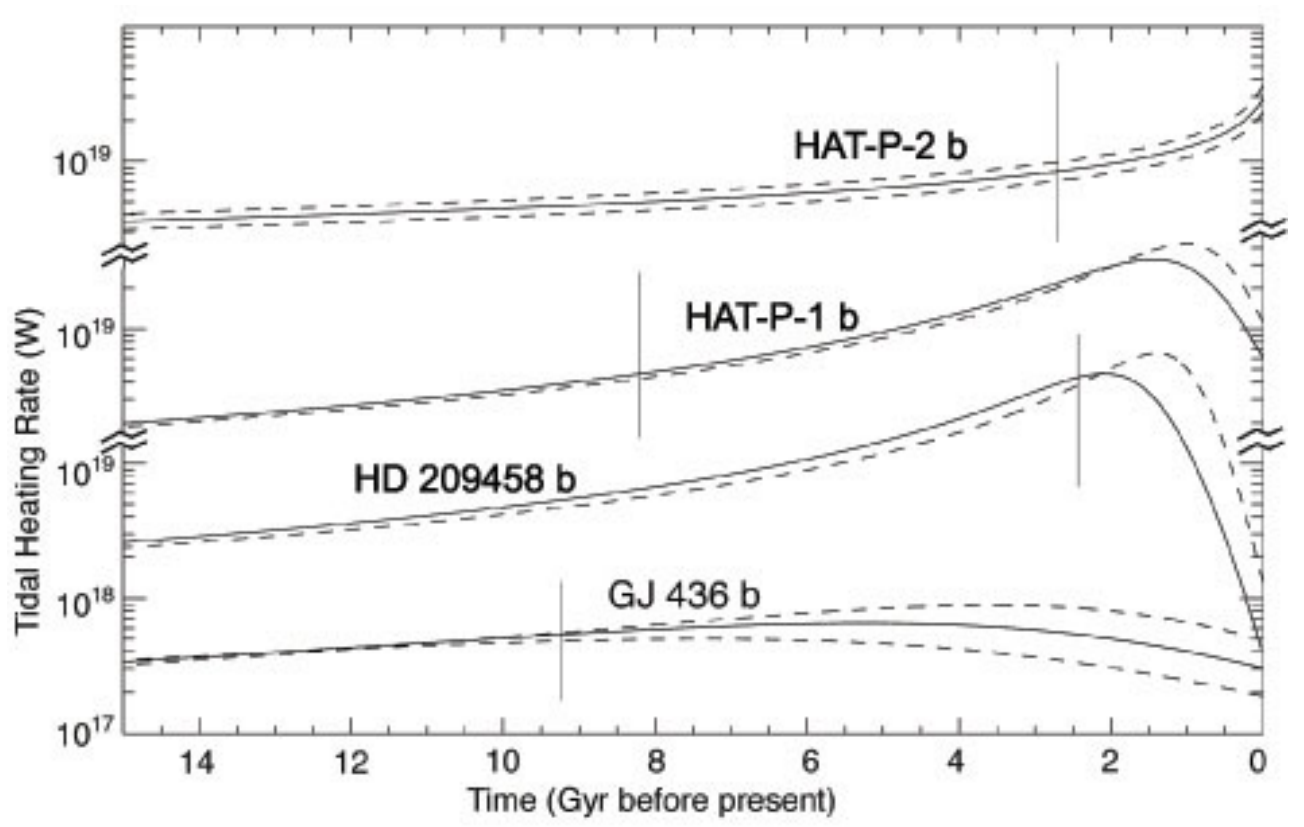

Figure 3. The tidal heating rates for planets HAT-P-2 b, HAT-P-1 b, HD 209458 b, and GJ 436 $\mathrm{b}$ as a function of time. The present time $(t=0)$ is at the right. The solid vertical line through each of the heating curves represents the estimated time of formation for that planet. Note that the vertical scales has been shifted for HAT-P-2 b and HAT-P-1 b to make its curves more visible. (The vertical scale that corresponds to each curve is the scale intersected by that curve.) The solid curve for each planet is based on the current nominal eccentricity value. The dashed lines represent upper and lower limits on the heating, i.e. they assume the extreme values of current $a$ and $e$ consistent with observations that give the maximum and minimum current tidal heating rates, respectively. For HAT-P-1 b and HD 209458 b, observations could not exclude a current eccentricity of zero, so the lower bound on heating rates is formally zero. Hence in those cases only one dashed line is shown, representing the upper limit. 
discrepancy between the large observed planetary radii and the predictions of physical modeling.

In the case of HAT-P-2 b there also has been a substantial amount of tidal heating. The current heating rate is similar to the maximum rate attained by HD $209458 \mathrm{~b}$ and HAT-P-1 b, so again we might expect a larger radius than predicted by theory that ignored tidal heating. In this case, however, the measured radius is actually about $12 \%$ smaller than predicted (Bakos et al. 2007b). Thus there is a discrepancy between theory and observation even if tidal heating is neglected. The fact that there is likely a high rate of tidal dissipation makes the problem even worse. On the other hand, a key factor in the reconciliation may be that, while the current tidal heating rate is high and increasing, in the recent past the heating rate was much lower. HAT-P-2 b is still on the increasing part of the heating curve, which is unusual among planets considered here, most of which have passed their peaks. The fact that the heating rate was several times smaller a billion years ago than it is now may help explain the small radius.

Figure 3 also illustrates tidal heating histories for GJ $436 \mathrm{~b}$. This planet has a measured radius consistent with theoretical models, independent of tidal heating (Gillon et al. 2007). The tidal heating history shown in Figure 3 is consistent with that result. Compared with the previous two cases, the maximum heating rate was two orders of magnitude less, small enough perhaps not to affect the radius.

\section{Discussion}

This investigation supports the hypothesis that tidal interactions between a star and a planet are responsible for the relatively small $e$ values of close-in planets (Rasio et al. 1996), although our calculations introduce important corrections to previous studies. Because even close-in planets evidently formed with much larger $e$, the processes that governed their early dynamics were probably similar to other planets. A plausible mechanism for producing the initial $e$ distribution of extra-solar planets is planet-planet scattering (Rasio \& Ford 1996; Weidenschilling \& Marzari 1996), although some modification to the original model are needed (Barnes \& Greenberg 2007).

If the tidal circularization hypothesis is borne out, then our study also provides constraints on $Q$ values. We find that agreement between eccentricity distributions requires $Q_{p} \sim 10^{6.5}$, with $Q_{*} \sim 10^{4.25}, 10^{5.5}$ (best fit), or $10^{6.75}$. This $Q_{p}$ is in good agreement with other constraints (Yoder \& Peale 1981; Ogilvie \& Lin 2004), and this $Q_{*}$ agrees well with studies of binary star circularization (Mathieu 1994). Of course, it is likely that individual planets and stars have unique $Q$ values, owing to variation in their internal structures. These $Q$ values are only meant to be representative values, good for the population as a whole. Corrections to our tidal model might also result in different suitable $Q$ values.

Significant reductions in semi-major axes have accompanied the changes in eccentricity, with important implications. First, models of protoplanetary migration in the primordial gas disk need not carry "hot Jupiters" in as far as their current positions. Lin et al. 1996 proposed that migration in the gas disk halted near the inner edge of the disk, a boundary determined by clearing due to the host star's magnetosphere. Our results show that the inner edge was probably farther out than indicated by the current semi-major axes of the planets, which were only reached during tidal migration long after the nebula 
had dissipated. In order to evaluate where migration due to the gas disk halted, (and thus where the inner edge of the nebula was) models should account for the subsequent tidal evolution.

The tidal changes in orbital semi-major axes also have implications for observations of planetary transits, such as surveys of young open galactic clusters (Bramich et al. 2005; von Braun et al. 2005; Burke et al. 2006). The probability to observe a planetary transit increases for smaller semi-major axes, but decreases as orbits become more circular (Borucki \& Summers 1984; Barnes 2007). Tidal evolution means that the probability of an observable transit depends on a star's age, but the exact relation depends on the particular evolutionary path through $(a, e)$ space. As our understanding of the statistics of tidal evolution paths improves, the observed frequency of transits in the field and in open clusters may eventually help to constrain planetary formation scenarios, distinguishing, for example, between the relative roles of embedded migration and of gravitational scattering, which set up the initial conditions for tidal evolution. Transit statistics may not yet be refined enough to be sensitive to detect this effect (Pepper \& Gaudi 2006), but such systematic effects may show up in future surveys.

The tidal heating calculations here suggest that past tidal heating may well have played an important role in the evolution of the physical properties of many extra-solar planets, specifically the planetary radius. We caution that the specific calculations displayed here depend on numerous assumptions and several uncertain parameters. The heating rates correspond to the orbital evolution trajectories computed by Jackson et al. 2008a, and various caveats are discussed in detail there. It is quite likely that the actual thermal history of any particular planet was different to some degree from what we show here. In particular, when analyzing radial-velocity observations to solve for a planet's orbit, it is difficult to rule out a completely circular orbit, in which case the tidal heating rate would have been zero. However, the unavoidable point is that past tidal heating may be significant for many planets and should be considered as a factor in theoretical modeling of physical properties of exoplanets.

For every planet whose tidal evolution we modeled, we have calculated corresponding tidal heating histories (Jackson et al. 2008b). For the cases presented here, see Fig. 3, we see that past tidal heating may provide a previously unconsidered source of heat for planets with larger-than-predicted radii. However, it may make things worse in cases where measured radii seemed to fit the current models. Theoretical models of tidal evolution and planetary interiors will generally need to be adjusted and improved so as to yield a match between predicted and observed planetary radii.

To conclude, we find that the distribution of orbital eccentricities for exoplanets was once strikingly uniform across all semi-major axes. By varying tidal dissipation parameters, we can match the original distribution of close-in planetary eccentricities to that of planets far from their host star for stellar and planetary $Q$ 's $\sim 10^{5.5}$ and $\sim 10^{6.5}$, which are consistent with previous estimates. After the formation of the close-in exoplanets, tides raised on the host star and on the planet acted over Gyrs to reduce orbital eccentricities and semi-major axes of the close-in exoplanets. This reduction in $e$ as well as $a$ has important implications for the thermal histories of close-in exoplanets and for transit studies.

We also find that tidal heating in the past was significantly larger than current heating. For example, about 1 Gyr ago, HD 209458 b may have undergone tidal heating 100 times the present value. This substantial heating may help resolve the mystery of the anomalously large radii observed for many transiting planets today. If the lag in response of the planetary radii to tidal heating is of order a Gyr, then past tidal heating must be 
included in models of exoplanetary radii. Previous studies suggest such a lag is reasonable. However, further studies are required to elucidate this effect.

\section{References}

Bakos, P., et al. 2007a, ApJ, 656, 552 .

Bakos, P., et al. 2007b, ApJ, 670, 826 .

Barnes 2001, ApJ, 561, 1095.

Barnes, R. \& Greenberg, R. 2007, ApJL, 659, L53.

Barnes, J. 2007, PASP, 119, 986.

Bodenheimer, P., Laughlin, G., \& Lin, D.N.C 2003, ApJ, 592, 555.

Borucki, W. J. \& Summers, A. L. 1984, Icarus, 58, 121.

Bramich, D. M. et al. 2005, Mon. Not. R. Astron. Soc., 359, 1096.

Butler, R. P. et al. 2006, ApJ, 646, 505.

Burke, C. J. et al. 2006, ApJ, 132, 210.

Burrows, A., Hubeny, I., Budaj, J., \& Hubbard, W. B. 2007, ApJ, 661, 514.

Da Silva, R. et al. 2006, A\&\&A, 446, 717.

Deming, D. et al. 2007, ApJL, 667, L199.

Fischer, D. \& Valenti, J. 2005, ApJ, 622, 1102.

Ford, E. \& Rasio, F. 2006, ApJ, 638, L45.

Gillon, M. et al. 2007, A\&A, 472, L13.

Goldreich, P. \& Nicholson, P. 1981, Icarus, 30, 301.

Goldreich, P. \& Soter, S. 1966, Icarus, 5, 375.

Gorda, S. \& Svechnikov, M. A. 1996, Astron. Reports, 42, 793.

Guillot, T. 2005, Ann. Rev. Earth and Planet. Sci., 33, 493.

Hubbard, W. B. 1984, Planetary Interiors, (New York: Van Nostrand Reinhold Co).

Jackson, B., Greenberg, R., \& Barnes, R. 2008a ApJ, accepted

Jackson, B., Greenberg, R., \& Barnes, R. 2008b, ApJ, submitted.

Johnson, J. A. et al. 2006 ApJ, 652, 1724.

Kaula, W. 1968, An Introduction to Planetary Physics, Wiley, NY.

Knutson, H., Charbonneau, D., Noyes, R., Brown, T., \& Gilliland, R. 2007, ApJ, 655, 564.

Laughlin, G. et al. 2005 ApJL, 629, L121.

Lin, D. N. C. et al. 1996, Nature, 380, 606.

Lovis, C. et al. 2006 Nature, 441, 305.

Maness, H. L. et al. 2007, PASP, 119, 90.

Mardling, R. 2007, Mon. Not. R. Astron. Soc., in press.

Weidenschilling, S. J. \& Marzari, F. 1996, Nature, 384, 619.

Mathieu, R. 1994, Annu. Rev. Astron. Astrophys., 32, 465.

Mayor, M. et al. 2004, A\&A, 415, 391.

McArthur, B. et al. 2004, ApJL, 614, L81.

Moutou, C. et al. 2006, A\&A, 458, 327.

Ogilvie, G. \& Lin, D. N. C. 2004, ApJ, 610, 477.

Peale, S. J., Cassen, P. \& Reynolds, R. T. 1979, Science, 203, 892.

Pepper, J. \& Gaudi, B. S. 2006, Acta Astronomica, 56, 183.

Rasio, F. A. \& Ford, E. B. 1996, Science, 279, 954.

Rasio, F. A., Tout, C. A., Lubow, S. H., \& Livio, M. 1996, ApJ, 470, 1187.

Rivera, E. et al. 2005, ApJ, 634, 625.

Saffè, C. et al. 2006, AछA A, 443, 609.

Takeda, G. et al. 2007, ApJS, 168, 297.

Trilling, D. 2000, ApJL, 537, L61.

Udry, S. et al. 2002, ApJ, 634, 625. 
Valenti, J. \& Fischer, D. 2005, ApJ, 159, 141.

Vogt, S. et al. 2005, ApJ, 632, 638.

von Braun, K. et al. 2005, PASP, 117, 141.

Wright, J. T. et al. 2007, ApJ, 657, 533.

Yoder, C. \& Peale, S. 1981, Icarus, 47, 1.

Zucker, S. et al. 2004, A\&BA, 426, 695. 